\title{
Nearshore Physical Oceanography: Recent Trends and Future Prospects
}

\author{
D.A. Huntley \\ Marine Institute, Plymouth University, Drake Circus, Plymouth, Devon, PL4 8AA, UK, D.Huntley@plymouth.ac.uk
}

\begin{abstract}
This paper reviews the evolution of nearshore physical oceanography over the past forty years. Over this period there have been enormous changes in the equipment, computing power and concepts used by nearshore physical oceanographers. The greatest change in observational equipment has been the development of remote sensing methods which are now providing spatial mapping of nearshore features and their temporal changes over time periods ranging from seconds to decades. The vast increase in computing power is also transforming the field from reliance on simplified analytical theories to comparisons with fully physical numerical simulations in realistic domains. Conceptually, the field has evolved from a Newtonian deterministic approach to a realization of the importance of the stochastic nature of the environment and of interactions between different aspects of the nearshore system.
\end{abstract}

\section{INTRODUCTION}

In this paper, I review, from a personal perspective, the evolution of nearshore physical oceanography over the past forty years, with a particular focus on identifying the major trends and continuing weaknesses, and trying to identify future prospects.

Enormous strides have been made over the past forty years in describing the nearshore physical environment, in understanding it and in making relevant predictions (or at least knowing the limitations of predictions) about future states. In order to survey these changes, it is useful to consider developments in three related areas: developments in equipment for observing the nearshore environment, developments in computing capacity available to the nearshore researcher and developments in concepts applied to the nearshore environment. These three areas are closely intertwined and complement each other in the evolution of our science, as we shall see.

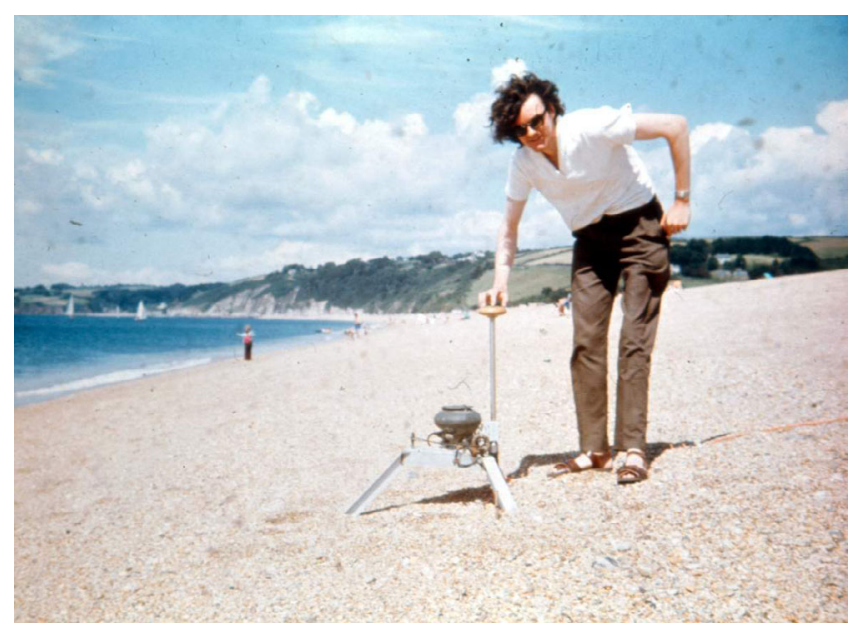

Figure 1. Waiting for high tide. An EM flowmeter (the discus under my hand) and a pressure sensor (the 'pot' on the aluminum frame) deployed in 1972.

\section{FORTY YEARS AGO}

The year 1972 was an auspicious year for nearshore physical oceanography! In that year, Jurjen Battjes published a seminal paper [Battjes, 1972] on the influence of the spectral nature of waves on the set-up of the water level at the shoreline, revealing in a startling way that the 'traditional' simplification of monochromatic, unidirectional waves approaching beaches could get things badly wrong. For example, for longshore currents, he showed that the directional spread of incoming waves could reduce the forcing by a factor of two.

It was also the year when electromagnetic current meters (EMs) were placed in the nearshore zone to measure, for the first time directly, the currents under waves as they shoal and break at the shoreline [Huntley and Bowen, 1975; figure 1]. Pressure sensors had been used for some time (including Barber and Ursell's observations of wave energy generated by storms half way across the world in the southern hemisphere [Barber and Ursell, 1948] and Tucker's observations [Tucker, 1950] of 'surf beat' from measurements of wave pressures off Peranporth in Cornwall, UK) but the addition of an ability to measure wave currents provided opportunities to interpret the wave motion in terms of wave directions and even to distinguish between wave types such as incident waves and edge waves and to begin to map out the flow patterns in and beyond the surf zone.

The resulting records of currents were immediately very interesting. The time series shown in figure 2, taken on that first deployment of the EM current meter, show clearly the change in the nature of the currents across the nearshore zone towards the shoreline, from the expected (though peaked) waves offshore to dominant longer period oscillations close to the shoreline. Comparisons of measurements on a steep beach and a beach of shallow slope also revealed important differences. As is usually the case when a new method of observation becomes available, the new means of in situ observation of nearshore hydrodynamics prompted a new burst of activity and new understanding of nearshore wave dynamics. 


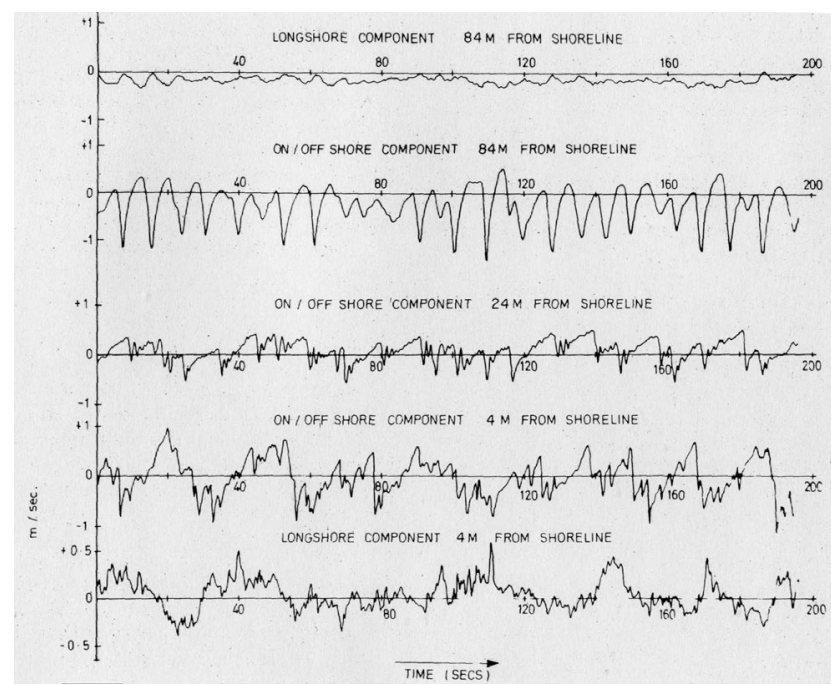

Figure 2. Current records from the first deployment of EM flow meters in the nearshore zone. The top two are the alongshore and cross-shore currents $84 \mathrm{~m}$ from the shoreline, the third the crossshore current $24 \mathrm{~m}$ from the shoreline and the lowest two the cross-shore and alongshore currents $4 m$ from the shoreline.

At the same time, the computing resources available to the researcher were growing rapidly but still very limited. The Institute where I worked in 1972 had only recently installed an IBM 1130 computer in a custom-built, air-conditioned room with a false floor to accommodate cabling. It boasted $64 \mathrm{kBytes}$ of core memory and a punched card reader for data and program input. Apart from running embryonic 2D tidal models, its main role in nearshore physical oceanography was as a data analyzer. A data set from the EM current meters could be input from magnetic tape but the programs to analyze it came in the form of a box of punched cards. These were presented to the computer before going home in the evening and, with luck, resulted in a printout of results the following day. Of course, any errors in programming meant punching new cards and waiting a further day for new results (a great way to encourage careful programming the first time round!). By this slow process, spectra of the EM-derived currents could be produced and wave contributions at different frequencies separated.

But the limited computing resources had another, more interesting influence. It meant that the interpretation of the measurements remained largely constrained to highly simplified, generally analytical solutions to the basic hydrodynamic equations. Thus the background theory of edge waves leading the interpretation of the long-period motion observed in the currents close to the shoreline was based on assumptions of very small amplitude waves (similar to Airy theory of waves), simple crossshore beach slopes (generally linear) and alongshore uniformity. Algorithms to compute the form of edge wave motion over arbitrary beach slopes (still alongshore uniform), and the realization that edge wave motion can be strongly sensitive to changes in cross-shore beach profile, didn't arrive for another six or so years. In the meantime, the knowledge that the simplified slopes were only an approximation provided a useful 'fudge factor' if the observations didn't quite fit the theory!
Forty years ago, the absence of sensors to measure short-term sediment response to waves, and the very limited role of computers in imposing realistic domains for wave motion, also meant that there was no challenge to the universal assumption that sediment moved in response to waves and currents and that any resulting patterns in beach morphology would reflect patterns in wave and current flow. Thus, the pursuit of edge waves in the natural environment was largely driven by an assumption that the form of cusps and nearshore bars reflected directly the flow patterns found in small amplitude edge waves over simple, unbarred topography.

This theoretical approach of relying on simplifications of the real physical environment and on simplifications of the basic hydrodynamic equations placed the field of nearshore physical oceanography forty years ago firmly in the 19th century tradition of reliance on analytical solutions to carefully chosen simplified situations. What was meant by increasing our understanding of the nearshore environment was centrally focused on linking observations to analytical solutions to simplified equation sets containing the key physical processes or processes responsible for given features. The underlying assumption was that if we got those processes right, the observed features would be 'explained' and potentially could be accurately predicted in a deterministic manner. As we will see, it is in this area of what we can expect as an 'explanation' for a phenomenon and the limits of predictability that the most radical, important and interesting changes have occurred over the last forty years.

\section{THE CURRENT STATE OF THE ART}

Let us now fast-forward to the present and see how the current state of the art compares to the situation forty years ago. Again here I will focus on the importance of equipment, computing and concepts as drivers of the radical changes that have occurred.

\section{Developments in sensors}

Of course, the first deployment of EM current meters was quickly followed by large-scale field campaigns where arrays of EM and other in situ sensors were deployed to measure the spatial structure of flows. The array of 42 sensors deployed over an area $520 \mathrm{~m}$ long parallel to the shore and $500 \mathrm{~m}$ offshore on Torrey Pines Beach, California in 1978 remains an outstanding example and allowed for the first time a clear demonstration of the expected spatial structure of long period edge waves [Huntley et. al. 1981], as well as providing data leading to a much better understanding of a whole range of nearshore processes, including the breaking of wave spectra [Thornton and Guza, 1983].

There have also been major advances in the development of sensors designed to measure sediment suspension by waves and currents. Point measurements by Optical Backscatter Sensors (OBSs) and acoustic sensors can now be complemented by acoustic sensors able to profile the suspended sediment concentrations over a range of depths. These sensors provide valuable insight into the complexities of sediment response to hydrodynamic drivers and have to some extent acted as constraints on the bewildering array of sediment transport algorithms on offer. It is probably fair to add, however, that this is still work in progress and a number of important factors remain unresolved. Chief amongst these factors are the difficulty of allowing for the strong dependence of sensor response on the size (and form, in the 
case of fine, flocculated sediments) of sediment particles, the need for very fine vertical resolution of the concentration profile close to the seabed, where concentrations are highest and the hydrodynamic regime most complex (see Austin and Masselink, [2008], for an example of the current state of the art), and the failure to date to find a workable means of measuring bedload transport at the seabed itself. Until these factors are better addressed our ability to improve our understanding of sediment transport under the full range of natural nearshore conditions remains limited.

More successful has been the development of sensors to monitor the evolution of bedforms in the nearshore zone. Acoustic sensors can now provide quantitative measurements of variations in bed elevation, including ripples and small sand waves [e.g. Austin et al. 2007] and are changing our perception of the nature of the seabed in the nearshore zone, not least in showing that bedforms persist even in the energetic surf zone.

The greatest recent advance in sensors for nearshore physical science has undoubtedly been in the development of methods of remote sensing of substantial areas of the nearshore zone. Most influential has been the Argus video-imagery system [Holman and Stanley, 2007], well known to anyone working in the field. The Argus system was originally set up to obtain maps of the locations and 2D-horizontal shapes of submerged nearshore bars through the breaking pattern over the shallower water over bars. The resulting time series showing the evolution of bars in response to forcing by offshore wave over periods of a year or more have been hugely influential in changing our perspective on bars, their dynamics and the processes responsible for forming them. In particular, the iconic 'movie' of a year in the life of bars on Palm Beach, Australia, produced by Rob Holman and his group at Oregon State University, could reasonably be called 'paradigmchanging' in its influence on the research community. No longer could simple ideas of a bar being the product of a single edge wave field be sustained; the temporal and spatial variability of the observed bars was clearly much more complex and there were strong hints that the evolution of bars depends as much on preexisting conditions as on the immediate forcing by waves.

Further development of the Argus technology has now led to its use not only for spatial mapping of topography, which can now

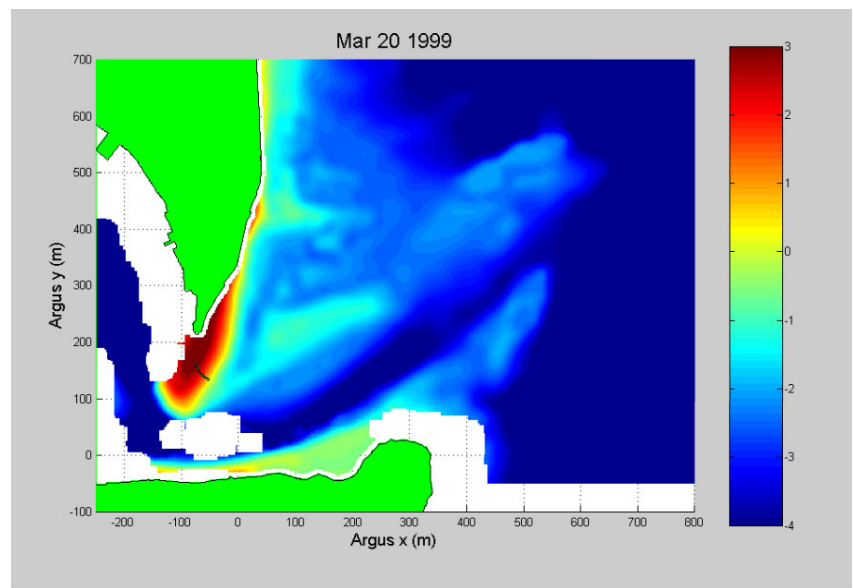

Figure 3. Bars at Teignmouth, Devon, UK. The bed elevations are given by the color bar to the right. These contours are derived from Argus images over several tidal cycles. With acknowledgement to Dr Kenneth Kingston, Plymouth University.
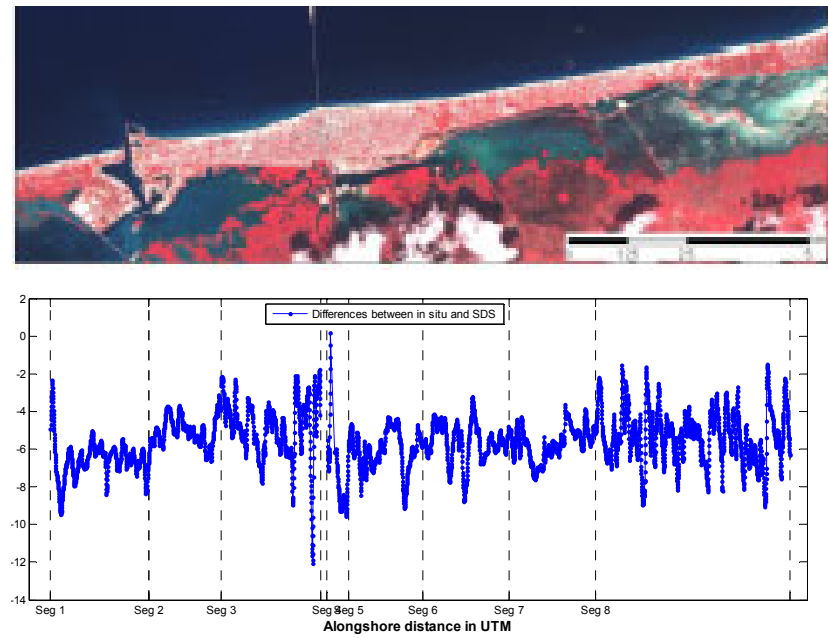

Figure 4. Upper panel: Satellite image of the northern coast of Yucatan, Mexico. Lower panel: The difference in position (meters) between the satellite-derived shoreline and the shoreline measured in situ using GPS. With acknowledgement to Gabriela Garcia Rubio, Plymouth University.

include quantitative estimates of water depths as well as horizontal shapes (figure 3), but also for estimation of wave directional spectra, wave refraction and nearshore currents [Holman and Stanley, 2007].

Satellites are also coming into use for evaluating nearshore processes, in particular showing their value in mapping, over large spatial scales, trends in shoreline erosion or accretion. Figure 4 shows an example of the accuracy of a satellite-derived shoreline along the north shore of the Yucatan Peninsula, Mexico, based on images from the SPOT satellite. The comparison with in situ measurements of the shoreline changes is very encouraging and confirms that the technique has great promise, particularly for regions with little or no other data on shoreline change.

By mapping, often in great detail, the spatial structure and temporal variation of nearshore morphology over a wide range of timescales, these new remote sensing techniques force the science of nearshore physical oceanography to confront the complexities of the real situation and expose the inadequacies of the simplified ideas which dominated the field forty years ago. Interpretation in terms of analytical solutions to greatly simplified scenarios no longer provides a satisfying explanation of the observed features. Fortunately, however, the concurrent development of computing power means that the need to rely on simplified cases is diminishing, as we show in the next section.

\section{Developments in computing}

It hardly needs to be said that computers have now come to dominate almost all aspects of life, not least in science. In our field, whereas forty years ago a computer was a tool for forecasting relatively simple hydrodynamic features such as tides and surges and for analyzing (slowly!) digital data from sensors, today computers impinge on almost all aspects of nearshore physical oceanography. In particular I want to emphasize three areas where computers have made, and continue to make, major changes to the pursuit of our science. Firstly computing power is 
now such that it can handle many of the complexities of the full equations of wave motion within realistic computational domains, thus removing the need for simplifying assumptions and simplified physical domains. Secondly it can be used to investigate the influence of complex, stochastic, boundary forcing and thus assess the predictability of the nearshore system. Thirdly it can include interaction between different features of the nearshore zone (waves and longshore currents; waves and nearshore circulation; waves and 2D topography and so on). Some examples of each of these areas will be given in this section.

Forty years ago, the numerical description of waves in the nearshore zone was generally limited to Airy theory, based on assuming sinusoidal waves with a single period and wavelength, and of a height much smaller than either the wavelength or the water depth. Today, computing power is beginning to allow computation of random wave shoaling and breaking using the full 3D Navier-Stokes equations, the only simplification being in the parameterization of turbulence by Reynolds averaging. These Reynolds-averaged Navier-Stokes (RANS) models can include all of the influences of realistic wave heights, non-linear wave distortion and wave breaking with none of the empirical input required by less complete models.

To date, application of RANS models to shoaling and breaking waves has been limited to shore-normal wave propagation with alongshore uniformity. The first such simulation was by Lin and Liu [1998] who successfully simulated 20s of data from a single breaking wave in the laboratory and this was followed by a simulation by Chopakatia et al. [2008] of wave propagation and breaking over the barred beach at Duck, North Carolina during the 1990 Delilah experiment. They simulated a 35.5 minute period, driving the outer boundary $800 \mathrm{~m}$ offshore with measured wave time series. The results are generally good, with wave heights predicted with rms errors of around $0.04 \mathrm{~m}$ and mean flows well predicted given the model assumption of alongshore uniformity. Wave breaking is directly included in RANS models and was identified in the model of Chopokatia et al. [2008] as the region where the maximum kinetic energy of turbulence exceeded a chosen threshold, with good agreement with observed breaking patterns in the field.

The computational requirements of RANS models will continue to limit their direct application to nearshore problem for some time to come, but their use to test different empirical parameterization used in simpler, less computer-intensive models has been demonstrated by Fuhrman et al. [2009] and Ruessink et al. [2009], who both tested the relative sensitivity of sediment transport under waves to the influences of wave height, velocity skewness, asymmetry and other variables, coming, intriguingly, to different conclusions about which influences are dominant!

Of more immediate value to the prediction of nearshore wave conditions are models which include non-linear effects but in simplified forms. Boussinesq theory relaxes the Airy theory assumption of infinitesimally small wave heights compared to water depth and wavelength and has been the subject of intensive research over the past forty years or more (for a review, see Huntley, in press]. Boussinesq models can now accurately model the propagation of waves from deep water right into the surf zone and in 2D domains, provided the slope of the seabed is small. These models do not inherently include wave breaking, which must be introduced empirically and there is still no consensus about how best this can be done [Cienfuegos et al., 2010]
Nevertheless, modern Boussinesq models can accurately simulate the heights of shoaling and breaking waves over 2D topography and include the skewness and asymmetry effects known to be vital to sediment transport. The focus now needs to shift to testing and tuning these models for nearshore sediment transport and morphological modeling.

As waves move into very shallow water, the assumptions of Boussinesq theory break down and the Nonlinear Shallow Water Equations (NSWE) become more relevant. As with Boussineq theory, these equations have been the subject of important recent developments [Brocchini and Dodd, 2008].

Remarkably, however, despite these developments, Airy theory continues to dominate practical methods for predicting nearshore wave conditions, despite the fact that nearshore waves generally grossly violate the assumptions of Airy theory, most obviously by having wave heights comparable to water depths and to wave wavelengths! This is in part explained by the fact that measurements have shown that Airy theory still provides a reasonable quantitative description of many features of nearshore waves (e.g. the relationship between wave height and wave currents; Guza and Thornton [1980]). Where Airy theory fails, empirical parameterizations can generally be patched in to cover the deficiencies. For example, to describe wave breaking through the surf zone, the earliest parameterization was to assume that breaking wave height is a fixed fraction of the local water depth, but this has generally now been replaced by more complex empirical descriptions of wave breaking in terms of roller dissipation and energy conservation. These parameterizations can be tuned to match a particular feature of the nearshore wave field, for example wave height through the surf zone, but such tuning can produce non-physical results for other features, for example the fraction of breaking waves in the surf zone (figure 5).

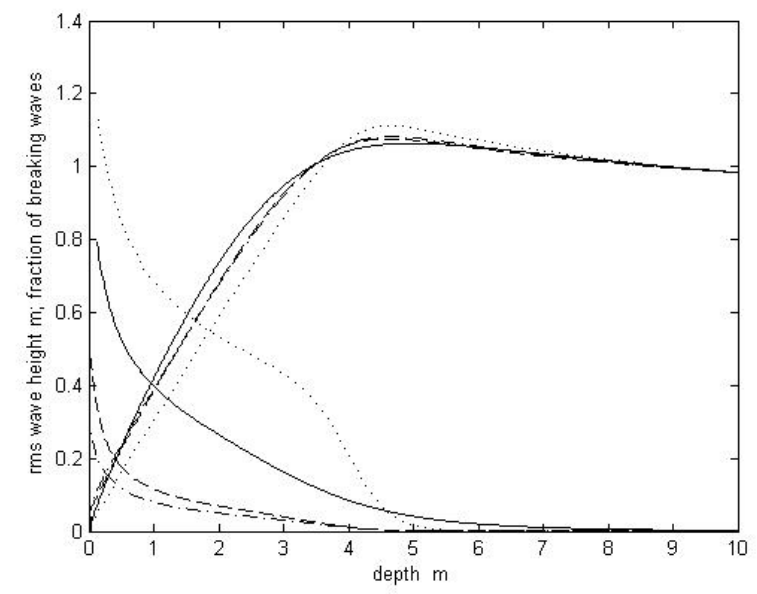

Figure 5. Predictions of shoaling and breaking of waves of $1 \mathrm{~m}$ deep water height and period 10s propagating over a beach slope of 0.01, for four breaking wave models. The curves reducing towards zero as the depth decreases show the modeled rms wave heights and curves increasing as depth decreases show the fraction of breaking waves. Solid line: Thornton and Guza [1983]; Dashed line: Battjes and Janssen [1978]; Dashed-dotted line: Baldock et al. [1996]; Dotted line: Lippmann et al. [1996]. Note that, despite the good match of wave heights, the predictions of the fraction of breaking differ widely and can exceed one. 
Nevertheless, the relatively small computational demands of wave models based on Airy theory mean that they continue to be the most used models in practice, despite their clear scientific limitations. The move to more sound physical models remains limited by computer power.

Whilst the move towards more complete physical models of nearshore waves is clearly valuable, another factor of equal, and arguably even more, importance is the fact that the forcing of a real nearshore system by incident waves is stochastic in nature. Random changes in the incoming wave field, whilst keeping integrated measures such as overall energy fluxes and directional spreads constant, can result in significant changes in the resulting response of the nearshore system. The possibility that system response might be sensitive to details of the incident forcing such as the sequence of varying wave heights (wave groupiness) and the chronology of storm-calm cycles, and that as a result predictability of future states in inevitably limited, has taken a surprisingly long time to enter the field of nearshore physical oceanography, particularly given that the seminal papers of Lorentz on non-predictability in meteorology were published as early as 1963 [Lorentz, 1963]. However the vastly increased computing power now makes it possible to make multiple model runs, each differing in the stochastic sequence of wave conditions, in order to assess sensitivities to this stochastic effect. Thus for example, de Vriend used an established morphodynamic model (Delft Hydraulics Unibest TC) to assess the sensitivity of bar evolution on the Dutch coast, over periods of 180 days and 10 years, to the chronology of storms incident on the coast (de Vriend: Presentation at NICOP Workshop, held at DHI, Copenhagen, June 1998). He concluded that the location and migration of the bars was relatively insensitive to stochastic variations but that the resulting bed elevations were chronologydependent and therefore much less predictable. Similar conclusions were reached by Coco et al. [2001] in their model for beach cusps.

The third aspect of numerical modeling that is now possible with current computing power is the ability to consider directly the interaction between different features such as waves and currents and waves and evolving topography and there is no doubt that this has had a profound influence on our understanding of nearshore processes. Here I will focus on the interaction between waves and the resulting morphodynamic response, as an example of the implications of this aspect.

As previously mentioned, forty years ago, the universal assumption was that the hydrodynamics of the nearshore zone determined the morphological response which creates beach profiles, bars, channels and shoreline cusps. The starkest demonstration of the inadequacy of this approach came in 1993 with the publication by Werner and Fink [1993] of their selforganization model for beach cusps. A disarmingly simple model for the hydrodynamics of swash motion and an even simpler model for sand response were coupled so that changes in the morphology fed back directly to the hydrodynamic response. The result was the emergence, due to self-organization within the coupled system, of beach cusps with a marked degree of alongshore regularity and a wavelength in good agreement with those observed in the field. The cusps emerged without any preexisting regular pattern in the flow field and thus the selforganisation model provided a completely different 'explanation' for cusps than the edge wave forcing model which had previously held sway. Subsequent detailed comparisons between observations and the self-organization model [Coco et al. 2000, 2001, 2003] have confirmed that the self-organization model is able to simulate a wide range of observed cusp characteristics, including growth to a quasi-equilibrium size and the observed variability in the alongshore spacing. It is now generally agreed that edge waves are not required for cusps to emerge and that they play only a minor role if they are present [Coco et al. 2001; Dodd et al., 2008].

Coupling between hydrodynamic and morphodynamic equations also plays a key role in the model used by Reniers et al. [2004] to simulate rip currents on an embayed beach. Their model produces a range of quasi-regular rip channels which are selforganizing when the incident waves have no directional spread but become primarily forced by quasi-steady flows generated by incident wave groups as the directional spread increases. Again, no correlation with simple edge wave wavelengths was found.

The success of the self-organization model of Werner and Fink [1993] is particularly extraordinary because of the simplicity of the hydrodynamic and sediment dynamic algorithms used. It appears that, at least for self-organized systems, inclusion of the feedback between water and sediment movement is overwhelmingly more important than getting details of the physics of either the water or sediment movement correct. This observation has led to a wide range of so-called 'abstracted models' which attempt to simulate a variety of nearshore (and other) morphological features (see Huntley et al. [2006], for a review). A major advantage of such models is that their demands on computational resources are relatively small so can be run many times with stochastically different forcing to provide an assessment of the intrinsic variability in their coupled systems.

\section{Developments in concepts}

The previous two sections have shown that the dramatic increase in resources available to the nearshore physical oceanographer have led to similarly dramatic changes in the way in which nearshore processes are perceived. Forty years ago the nearshore system was perceived through linear, Newtonian deterministic eyes, with hydrodynamic behavior decoupled from topographic response and a highly simplified view of incident wave forcing (typically unidirectional, monochromatic waves) and highly simplified descriptions of nearshore domains. The result was that an 'explanation' for any particular behavior was typically couched in terms of agreement with analytical solutions of simplified theoretical models which best fitted the limited observations available. We now have a much greater ability to observe large areas of nearshore systems and to track their evolution over a range of time scales. We can also now model numerically much more realistic computational domains with much more realistic physical processes. The result is a shift from explaining observations in terms of simplified, analytical results towards explanations based on agreement with numerical model outputs. Where models include details of the expected physical processes acting on the system, such an explanation might be in terms of the relative importance of the different physical processes which are included. For the highly simplified 'abstracted' models, however, emerging self-organized features are 'explained' by the strong coupling and feedback included in the model rather than any particular physical process, an interesting and fundamental change in perception of the meaning of 'explanation'! 
There is also a much greater (though perhaps still not universal) appreciation of the intrinsic limits to the predictability of the nearshore environment. As we have seen, some features (for example some mean length scales of morphological features) have been found to be relatively insensitive to the stochastic nature of wave forcing but others (for example the bed elevations associated with morphological features) are much more unpredictable. The need to address such limits to predictability is also an important recent conceptual change.

\section{FUTURE PROSPECTS}

This brief and personal view of the evolution of nearshore physical oceanography over the past forty years does suggest some trends which might be expected to continue and develop into the future.

In terms of equipment development, notwithstanding the insensitivity of many morphological features to details of sediment transport, there remains an outstanding need for better in situ sensors to measure sediment in motion in the nearshore zone. Sediment transport algorithms can currently only be tested against limited and incomplete data sets and are therefore uncertain and limited to narrow ranges of environmental conditions. It is unfortunately difficult to see where major advances are likely in this area of research but combinations of optical and acoustic techniques probably still form the best available approach. Remote sensing techniques for the nearshore environment are also still being developed and will continue to be central to the development of research in the future.

It is obvious that numerical modeling will continue to increase in importance for providing explanations and predictions of nearshore behavior. However there are several directions that use of increased computing power could go. Improving the physical description of relevant physical processes will take up more computing time. For example have seen that there is a hierarchy of available numerical models for nearshore waves and it is clearly desirable to move increasingly towards those which include better, less empirical, descriptions of fundamental processes. Alternatively any increase in computing power could be used to improve the coupling between nearshore features. As we have seen, it is now clear that coupling between hydrodynamics and morphological response is vital if at least some nearshore phenomena are to be properly understood and simulated. Coupled models however demand more computer time than decoupled models, either through the need for concurrent running of interacting equation sets or through the use of more complex equations. A third possibility would be to use additional computing power to assess sensitivity to the stochastic forcing that is the natural feature of a nearshore system. We have seen that some nearshore features are particularly sensitive to forcing characteristics such as the detailed form of incident wave groupiness or the chronology of storms.

Of course there is not a single best answer to the question of where increased computing power should be directed. The optimum approach will vary depending on the particular nearshore feature of interest and research will continue in all three directions. However, it is important to recognize that there is a choice to be made.

For example, the surprising success of abstracted models in reproducing many of the features of at least some quasi-regular morphological features in the nearshore zone (cusps, large-scale longshore bars, ripples, sorted bedforms) suggests that improvements in the physical processes should be included in the models only as they reveal deficiencies in simulating particular features. For beach cusps, for example, changes to the sediment transport algorithm are found to influence the rate at which cusps grow but have only a small influence on the length scales and equilibrium relief of the cusps. In this case use of computing power to investigate sensitivity to changing wave forcing and to pre-existing morphological conditions might be more appropriate.

Finally it is worth pointing out that models to simulate nearshore behavior have now developed to the point where they can begin to be used as research tools in their own right. We have already seen the example of the use of RANS models by Fuhrman et al. [2009] and Ruessink et al. [2009] to investigate the relative importance of different processes in determining the response of sediment to waves, with a view to providing better empirical descriptions for simpler models. Huntley et al. [2008] show how an abstracted model of ripples can be used to investigate how a ripple field responds (or not) to a change in the current field above them. It is likely that such numerical 'thought experiments' will be increasingly common in the future.

\section{REFERENCES}

Austin, M. J and G. Masselink (2008). The effect of bedform dynamics on computing suspended sediment fluxes using optical backscatter sensors and current meters. Coastal Eng. 55(3), 251-260.

Austin, M. J., G. Masselink, T. J. O'Hare and P. E. Russell (2007). Relaxation time effects of wave ripples on tidal beaches. Geophys. Res. Let. 34, L16606, doi:10.1029/2007GL030696

Baldock, T. E., P. Holmes, S, Bunker and P. van Weert (1998). Cross-shore hydrodynamics within an unsaturated surf zone. Coastal Engineering 34(3-4): 173-196

Barber, N. F. and F. Ursell (1948). The generation and propagation of ocean waves and swell: 1. Wave periods and velocities. Phil. Trans. Roy. Soc. London, Series A; Mathematical and Physical Sciences, 240 (824), 527-560.

Battjes, J. A. (1972). Set-up due to irregular waves. Proceedings of the 13th International Conference of Coastal Engineering. New York, ASCE

Battjes, J. A. and J. P. F. M. Janssen (1978). Energy loss and setup due to breaking of random waves. 16th International Conference on Coastal Engineering, Hamburg, ASCE, New York.

Brocchini, M. and N. Dodd (2008). Nonlinear shallow water equation modeling for coastal engineering. Journal of Waterway, Port, Coastal and Ocean Engineering 134(2): 104120

Chopakatia, S. C., T. C. Lippmann and J. E. Richardson (2008). Field verification of a computational fluid dynamics model for wave transformation and breaking in the surf zone. Journal of Waterway, Port, Coastal and Ocean Engineering 134(2): 71-80.

Cienfuegos, R., E. Barthelemy and P. Bonneton (2010). Wavebreaking model for Boussinesq-type equations including roller effects in the mass conservation equations. Journal of Waterway, Port, Coastal and Ocean Engineering 136(1): 10-26. 
Coco, G., T. K. Burnet, B. T. Werner and S. Elgar (2003). Test of self-organisation in beach cusp formation. J. Geophys. Res., 108(C3), 3101, doi: 10.1029/2002JC001496.

Coco, G., D. A. Huntley and T. J. O'Hare (2000). Investigation of a self-organised model for beach cusp formation and development. J. Geophys. Res. 105, 21,991-22,002.

Coco, G., D. A. Huntley and T. J. O'Hare (2001). Regularity and randomness in the formation of beach cusps. Marine Geology 178: $1-9$.

Dodd, N., A. Stoker, D. Calvete and A. Sriariyawat (2008). On beach cusp formation. Journal of Fluid Mechanics 597: 145169.

Fuhrman, D. R., J. Fredsoe and B. M. Sumer (2009). Bed slope effects on turbulent boundary layers: 2. Comparison with skewness, asymmetry and other effects. Journal of Geophysical Research-Oceans 114(C03025).

Guza, R. T. and E. B. Thornton (1980). Local and shoaled comparisons of sea surface elevations, pressures and velocities. Journal of Geophysical Research 85(C3): 1524-1530.

Holman, R. A and J. Stanley (2007). The history and technical capabilities of Argus. Coastal Engineering, 54 (6-7), 477-491.

Huntley, D. A. (in press). Nearshore Processes: Waves. in.: Shroder, I. (Ed-in chief), Sherman, D. (Ed.) Treatise on Geomorphology. Academic Press, San Diego. Vol. 10.

Huntley, D. A. and A. J. Bowen (1975). Comparison of the hydrodynamics of steep and shallow beaches. Chapter 4 in: Nearshore Sediment Dynamics and Sedimentation. Hails, J and Carr A. (Eds). John Wiley and Sons, London.

Huntley, D. A., G. Coco and T. J. O’Hare, (2006). Abstracted modelling as a tool for understanding and predicting coastal morphodynamics. J. Coastal Res. SI49, 21-27. Proceedings of ICS2004, Itajai, Brazil.

Huntley, D. A., Coco, G., K. R. Bryan and A. B. Murray. (2008). Influence of "defects" on sorted bedform dynamics. Geophys. Res. Let. 35, L02601. doi: 10.1029/2007GL030512

Huntley, D. A., R. T. Guza, and E. B. Thornton. (1981). Field observations of surf beat, 1. Progressive edge waves. Journal of Geophysical Research 86(C7): 6451-6466.

Lin, P. and P. L. F. Liu (1998). A numerical study of breaking waves in the surf zone. Journal of Fluid Mechanics 359: 239264.

Lippmann, T. C., A. H. Brookins and E. B. Thornton (1996). Wave energy transformation on natural profiles. Coastal Engineering 27: 1-20

Lorentz, E. N. (1963). Deterministic non-periodic flow. J. Atmos. Sci. 20, 131-141.

Reniers, A., E. B. Thornton and J. A. Roelvink (2004). Morphodynamic modeling of an embayed beach under wavegroup forcing. Journal of Geophysical Research 109(C01030).

Ruessink, B. G., T. J. J. van den Berg and L. C. van Rijn (2009). Modeling sediment transport beneath skewed asymmetric waves above a plane bed. Journal of Geophysical Research-Oceans 114(C11021)

Thornton, E. B. and R. T. Guza (1983). Transformation of wave height distribution. Journal of Geophysical Research 88(C10): 5925-5938

Tucker, M. J. (1950). Surf beats: Sea waves of 1 to 5 min. period. Proceedings of the Royal Society Series A 202: 565-573

Werner, B. T. and T. M. Fink (1993). Beach cusps as selforganised patterns. Science 260: 968-971. 